1388

\section{ASSESSMENT OF A SERIES OF MARKERS AS DIAGNOSTIC TOOLS OF EARLY-ONSET NEONATAL SEPSIS}

\author{
G. Zaharie ${ }^{1}$, M. Popa 2 , S. Bolboaca ${ }^{3}$, L. Blaga ${ }^{2}$,
} N. Schmidt ${ }^{2}$, M. Matyas ${ }^{2}$, T. Zaharie $^{4}$

${ }^{1}$ Neonatology, University of Medicine and Pharmacy Iuliu Hatieganu Cluj, ${ }^{2}$ Neonatology,

${ }^{3}$ Informatics and Biostatistics, University of

Medecine and Pharmacy Iuliu Hatieganu Cluj-

Napoca, ${ }^{4}$ Pathology, County Hospital, Cluj-

Napoca, Romania

Aim: The aim of the research was to evaluate the usefulness of Toll-like receptors 2(TLR-2) and 4(TLR-4), interleukine-6(IL-6), tumor necrosis factor-alpha(TNF- $\alpha$ ) and C-reactive protein(CRP) in diagnosis of early neonatal sepsis on preterm babies with premature rupture of membranes.

\section{Material and methods:}

A prospective study has carried out between jan.2007-jan.2008 at Neonatology Department of the Cluj District University Hospital.

Diagnosis of sepsis was done according to International Sepsis Definitions Criteria. Study group involved 21 newborns with symptoms suggestive of systemic infection, requiring sepsis evaluation, antibiotic treatment and control group represented by healthy newborns. We determined in the first day of life TLR-2, TLR-4, IL-6, TNF- $\alpha$ and CRP and in the third day the same without TLR. We used latex agglutination test for CRP, Elisa technique for TNF- $\alpha$ and IL-6 and flow cytometry for TLR. Data were analyzed using Statistica 6.0.

Results: Sepsis group presented in the 1st day: TNF- $\alpha \quad(p g / m l)=14,7[5,0-24,3]$; IL6 (pg/ $\mathrm{ml})=153,7[82,3-225,1] ; \quad$ CRP $(\mathrm{mg} \%)=0,83[0,54-$ $1,12]$; and the expression(\%) of TLR2 $=42,5$ [29,555,4]; and TLR4 $=2,2[1,26-3,15]$. TNF- $\alpha$ was correlate significantly and negative with TLR2. TLR2 was correlated significantly and positive with TLR4. In the 3-rd day the values were: TNF- $\alpha(\mathrm{pg} / \mathrm{ml})=$ $10,1[5,1-15,1] ; \quad$ IL6 $(\mathrm{pg} / \mathrm{ml})=46,5[16,3-76,7] ; \quad$ CRP $(\mathrm{mg} \%)=1,2[0,6-1,81]$. Control group presented: $\operatorname{TLR} 2(\%)=5,69(p=0,00006)$ and $\operatorname{TLR} 4(\%)=$ $0,67(p=0,037)$.

Conclusions: IL- 6 and TNF- $\alpha$ can be considered markers of early-onset neonatal sepsis. TLR-2 and TLR-4 could be promising markers for neonatal sepsis. CRP revealed not to be a useful marker in diagnosis of early-onset sepsis.

\section{9}

\section{IMPACT OF MATERNAL SEXUAL HABITS AND HYGIENE BEHAVIOURS ON GBS NEONATAL COLONIZATION}

J. Buinauskiene ${ }^{1}$, R. Nadisauskiene ${ }^{2}$,

R. Tameliene ${ }^{1}$, E. Barcaite ${ }^{2}$, D. Stoniene ${ }^{1}$, E. Markuniene ${ }^{1}$, A. Vitkauskiene ${ }^{3}$

${ }^{1}$ Neonatology, ${ }^{2}$ Obstetrics and Gynaecology, ${ }^{3}$ Microbiology, Kaunas University of Medicine, Kaunas, Lithuania

Background and aims: Early onset neonatal sepsis due to vertical transmission of Group B streptococci (GBS) is responsible for severe morbidity and mortality of newborns. The aim of study was to determine the prevalence of neonatal GBS colonization and to evaluate the impact of maternal sexual habits and hygiene behaviours on neonatal GBS colonization.

Methods: A prospective cross-sectional study was carried out at the Departments of Obstetrics \& Gynecology and Neonatology Kaunas University Hospital in 2006-2007. Data of 970 women and 827 newborns were analyzed. A lower vaginal and rectal swab were obtained from each women at 3537 weeks of gestation or on admission for PROM or at delivery. Cultures of neonates were sampled from the ear canal and throat within 5-15 min of their lives. Study participants were asked to complete a questionnaire described their sexual habits and hygiene behaviours.

Results: Overall 148 (15.3\%) women were carriers of GBS. Neonatal GBS colonization rate was $6.4 \%$. Overall vertical transmission rate of GBS was $28.4 \%$. Univariate analysis revealed that neonatal GBS colonization was associated with oral sex male-tofemale (60.0 vs $36.3 \%, p<0.001)$, female-to-male (56.0 vs $38.2 \% p<0.05$ ), male not douching before sex $(8.0$ vs $0.9 \%, p<0.001)$. The other maternal sexual habits like condom use, anal sex, number of sexual partners, time of first sexual experience, hand washing frequency were not significantly associated with neonatal GBS colonization.

Conclusions: We consider that changing of sexual habits and improvement of hygiene behaviours could help to facilitate the neonatal GBS colonization. 